\title{
The Scaling of Attention Networks
}

Cheng-Jun Wang ${ }^{1}$, Lingfei $\mathrm{Wu}^{2 *}$

1 School of Journalism and Communication, Nanjing University, Nanjing 210093, P.R.

China

2 Center for Behavior, Institutions and the Environment, Arizona State University, Tempe, AZ 85281, U.S.

* E-mail: wlf850927@gmail.com

\begin{abstract}
We use clicks as a proxy of collective attention and construct networks to study the temporal dynamics of attention. In particular we collect the browsing records of millions of users on 1,000 Web forums in two months. In the constructed networks, nodes are threads and edges represent the switch of users between threads in an hour. The investigated network properties include the number of threads $N$, the number of users $U V$, and the number of clicks, $P V$. We find scaling functions $P V \sim U V^{\theta_{1}}, P V \sim N^{\theta_{3}}$, and $U V \sim N^{\theta_{2}}$, in which the scaling exponents are always greater than 1 . This means that 1$)$ the studied networks maintain a self-similar flow structure in time, i.e., large networks are simply the scaleup versions of small networks; and 2) large networks are more "productive", in the sense that an average user would generate more clicks in the larger systems. We propose a revised version of Zipf's law to quantify the time-invariant flow structure of attention networks and relate it to the observed scaling properties. We also demonstrate the applied consequences of our research: forum-classification based on scaling properties.
\end{abstract}

\section{Introduction}

Attention economics was firstly proposed by H. A. Simon et al. in 1971 to address the conflict between the scarcity of human attention and the explosive growth of information [1]. In the past decades, with the development of media technology such as the World Wide Web and smartphone, it is increasingly easier to copy and distribute information, thus the task of developing a quantitative framework for attention dynamics becames more urgent. Fortunately, the availability of online data sets allows scholars to investigate the dynamics of attention empirically. For example, studies have been conducted to investigate the competition for user attention between emails [2], news [3], tags [4], and tweets [5 7]. A conclusion already drawn is that the competition usually leads to a long-tail distribution of attention allocated on information resources 2,8]. However, how this distribution evolves in time and how the evolution shapes the growth of online information systems remain unknown.

To address these questions, we collect clickstream data from a group of Web forums and use clicks as a proxy to study the dynamics of attention. We construct networks for each of the top 1,000 forums in Baidu Tieba, a very large Chinese online system containing many forums of diverse topics. In these networks, nodes are threads and edges represent "user-flow", i.e., the switch of users between threads in an hour. We call these networks "attention network" because they show how the collective attention of users is transported between threads within a forum. Several limitations of our study should be noted. Firstly, using clicks as an approximation of attention helps us focus on the most essential and traceable activity in the cyber-world, but the information on the duration of visits is lost. Therefore, we have to assume that all threads that receive the same number of clicks are equally popular. Another limitation is that we only study the clickstreams between threads in forums, which are not constrained by the tree structure of posts within threads, but are still strongly affected by the displaying order of threads on the homepage of forums.

We find that these networks exhibit the same scaling properties as discovered in real-world flow systems 
including rivers 9 and circulatory systems $[10$. These scaling properties are usually viewed as a signature of the time-invariant flow structure [11. We propose a revised version of Zipf's law to describe the effect of system size on distribution function in flow systems. We find that the discussed scaling properties can be derived from such a function, which means that the discovered hidden connection between size and structure may widely exist in various flow systems.

These findings help us understand why we may spend more time on larger forums: most of the studied attention networks preserve a self-similar flow structure such that when it becomes larger, it is more difficult for a random walker to leave from the system. This pattern is not only interesting by itself, but also has strong applied consequences. As a demonstration we group the studied forums into 20 categories according to their topics and try to relate the discussed self-similar flow structure with the topics of forums. It turns out that the forums in the "Cities" category are the most attractive, whereas the "Art" forums are the least attractive. Our clickstream-based analysis not only provides development suggestions for the Tieba system, but also presents a general method that can be easily applied to many other online social systems.

\section{Materials and Methods}

\section{Data source}

Baidu (http://www.baidu.com/), a leading Chinese language search engine, is the fourth largest site in the world according to Alexa (http://www.alexa.com/topsites). Tieba (http://tieba.baidu.com/) is a big forum systems supported by Baidu. In this system, users search a forum by typing a keyword within the system, and if the forum has not been created yet, it will be created upon the search. This novel design successfully kept search traffic within the wbesite and effectively motivated users to contribute. As of 2014 more than eight million forums are created (mostly by fans). The topics of these forums cover popular stars, movies, comics or books. More than one billion threads are created in these forums.

In our research we collect the browsing activities of users on the top 1,000 forums, whose daily traffic (the number of unique visitors) varies from thousands to millions. For each forum, we construct 1,440 successive hourly-based attention networks in two months (from Feb. 27, 2013 to Apr. 27, 2013). See Figure 1 for the construction of attention networks in details.

\section{Clickstream networks and key variables}

Figure 1 presents an example Baidu Tieba clickstream network, whose nodes are threads and edges represent the switch of users between threads. In the annotation of Figure 1 we introduce how to construct clickstream networks from users' browsing records. We firstly divide the entire data set into hourly pieces and then sort each piece by cookies (the unique and permanent labels used by a website to identify users). After that, we select all successive pairs of threads visited by the same user and add corresponding edges in the clickstream network. Sorting data by cookies guarantees that a user would not be repeatedly counted even if the he/she entered and left the system more than once during an hour.

After two artificial nodes "source" and "sink", which represent the "environment", are added to the clickstream networks, they satisfy "flow conservation", i.e., inflow equals outflow both on the network level and also on the node level. As a result, the network properties $P V$ (the total number of clicks) and $U V$ (the total number of users) can also be expressed as the sum of flow passing through node $i$ ( $\left.T_{i}\right)$ and the sum of flow dissipated by node $i$ to "sink" $\left(D_{i}\right)$, respectively:

$$
U V=\sum_{i=1}^{N} D_{i}
$$




$\begin{array}{lll}\text { a } & 0 & \\ \text { a } & 1 & \text { A } \\ \text { a } & 2 & \\ \text { b } & 0 & \\ \text { b } & 2 & \\ \text { c } & 2 & \\ \text { c } & 3 & \\ \text { d } & 0 \\ \text { d } & 4 \\ \text { d } & 5 & \\ \text { e } & 5 & \\ \text { e } & 0 & \end{array}$

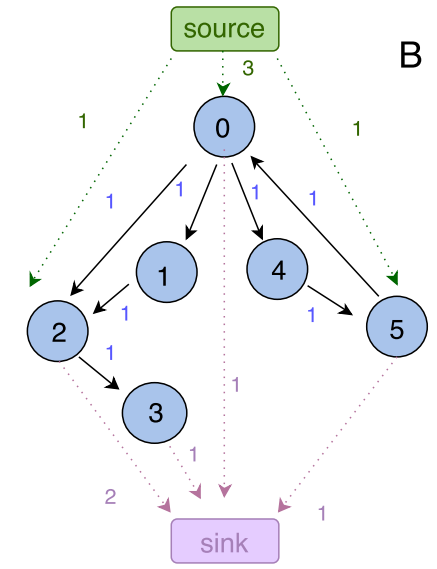

B

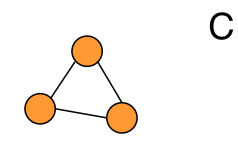

C

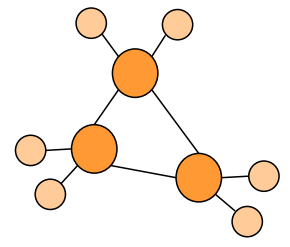

Figure 1. An example of Baidu Tieba log file and the corresponding clickstream network. In Panel A the left column shows the anonymized, sorted cookies and the right column shows the numeric IDs of the visited threads. In Panel B the nodes are threads and the weighted, directed links represents clickstreams, i.e., users' switch between threads. The green arrows show the clickstreams receive by nodes from "source" and the purple arrows show the clickstreams dissipated to "sink". In particular, the network in Panel B is constructed as follows. For each record in the dataset, say, [a, 0], if the next record has the same cookie, e.g., [a, 1], we add a clickstream from node 0 to node 1 ; otherwise, we create a clickstream from node 0 to the artificially added node "sink". After all records are converted into clickstreams, we add a "source" node to balance the network such that in-flow (weighted in-degree) equals out-flow (weighted out-degree) over all nodes except "source" and "sink". In the constructed networks, the values of passing-through clickstreams $T_{i}$ from node 0 to 5 are $\{4,1,3,1,1,2\}$, and the values of corresponding dissipation $D_{i}$ are $\{1,0,2,1,0,1\}$. The values of $P V$ and $P V$ of this network are 12 and 5, respectively. The value of $P V$ equals the sum of $T_{i}$ and the value of $U V$ equals the sum of $D_{i}$, respectively, as suggested by Eq. 1 and Eq. 2. Panel C gives a naive example to show how an attention network preserves self-similarity over time.

$$
P V=\sum_{i=1}^{N} T_{i}
$$

\section{Results}

\section{The scaling functions of forums}

We find that the number of clicks $(P V)$ scales to the number of unique visitors $(U V)$ during the growth of forums, satisfying

$$
P V \sim U V^{\theta_{1}},
$$

as shown in Fig 11. The scaling exponent $\theta_{1}$, which reflects the ratio of relative increase of clicks over the relative increase of users, is greater than unity and strikingly remains as a forum-specific constant during the studied time period (1,440 hours). The observed scaling function means that 1$)$ the studied forums exhibit self-similarity structure in growth such that the well-developed forums are merely scaleup versions of those in early stages; 2) the average surfing length increases with user population, i.e., 

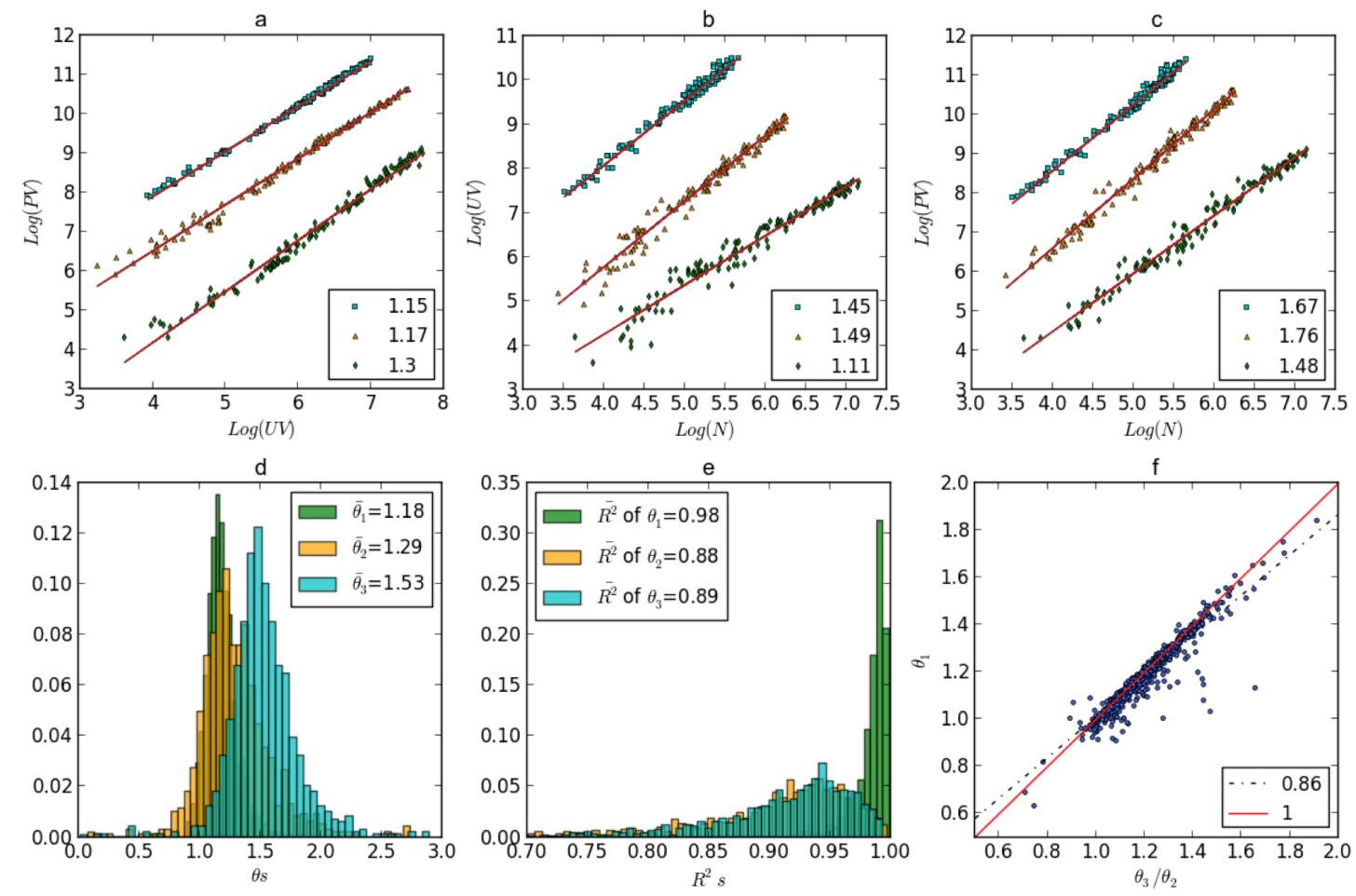

Figure 2. The scaling function of forums. Panel $\mathrm{A} \sim \mathrm{C}$ show the scaling relationships between $P V$ (the number of clicks), $U V$ (the number of users), and $N$ (the number of threads) across three forums in two months $(1,440$ hours). Data points are hourly-based and the statistics of different forums are plotted in different colors and shapes. Panel D $\sim$ F give the result of fitting Eq $3 \sim$ Eq 5 by empirical data. Panel $\mathrm{D}$ shows the distribution of the estimated scaling exponents. Panel $\mathrm{E}$ shows the distribution of the values of $R^{2}$ in the estimation. In Panel $\mathrm{F}$ we validate Eq.6. In Panel $\mathrm{F}$, we plot the regression line given by orthogonal regression (the black, dotted line) as well as the theoretical line predicted by Eq 6 .

$P V / U V \sim U V^{\theta_{1}-1}$, exhibiting the property of economics of scale. These two findings are not only interesting in theory, but also have applied relevance. First of all, website managers can use $\theta_{1}$ as an indicator to benchmark and monitor the productivity of forums, as a large $\theta_{1}$ means that an average user would generate more clicks in browsing threads. In Fig 3 we show that the forums of different topics are not equally productive. For example, the regional forums (the "Cities" category) are much more productive than the forums discussing arts (the "Art" category). Besides its applied value in forum management, $\theta_{1}$ can also be used as a novel feature to facilitate thread recommendation [12].

We find that, $\mathrm{Eq} 3$ can be broken into

$$
U V \sim N^{\theta_{2}},
$$


and

$$
P V \sim N^{\theta_{3}},
$$

which characterize the growth of users and clicks with respect to the number of threads $(N)$, respectively. And the connection between the two equations is given by

$$
\theta_{1}=\frac{\theta_{3}}{\theta_{2}} .
$$

Panel $\mathrm{A} \sim \mathrm{C}$ in Fig 1 show the empirical relationships expressed by Eq.3 $\sim \mathrm{Eq} .5$ i.e., the scaling relationships between the number of clicks (PV), the number of users (UV), and the number of threads (N) using the data sets of three different forums in the time period under study (1,440 hours). Note that a clear linear trend in a log-log scale is a characteristic signature of a power-law function. In Panel D and E we report the parameter distributions in fitting Eq $3 \sim \mathrm{Eq} 5$ using the data sets of all the studied 1,000 forums. We observe narrow, unimodal distributions of scaling exponents with mean values greater than 1 and left-skewed distributions of $R^{2}$ with mean values approximate 1 . It means that the scaling models fit empirical data very well, and most of the empirical scaling relationships are super-linear. Finally, the relationship between the exponents of the three scaling functions is empirically tested in Panel $\mathrm{F}$, which shows that $\theta_{1}$ is linearly depend on $\frac{\theta_{3}}{\theta_{2}}$ as predicted by Eq.6. All this is evidence that discussed scaling functions are universal properties of the studied Web forums.

At this stage, it is natural to ask how Eq4 and Eq 5 emerge as strong regularities that shape the evolution of attention networks. Inspired by previous studies on real-world flow systems [10], we conjecture that the observed scaling pattern can be explained by the self-similar structure of clickstream transportation. In the following section, we will quantify this topological self-similarity and explain how it gives rise to the observed scaling patterns.

\section{From revised Zipf's law to scaling functions}

In the last section we introduced how to construct clickstream networks that satisfy Eq1 and Eq2 If these networks preserve time-invariant structure as we assumed, i.e., the distribution of passing-through flow $T_{i}$ and dissipation $D_{i}$ on nodes does not change in time, we should be able to derive the scaling functions of $P V$ and $U V$ on $N$ from these distributions. But before this, we need to find the correct form of the distribution of $D_{i}$ and $T_{i}$.

Unsurprisingly, we observe that the hourly distributions of $D_{i}$ and $T_{i}$ have long-tails, which are widely observed in online social systems [13]. However, these distributions do not satisfy the traditional form of power-law distribution, or Zipf's law 14, but change with system size. Therefore, we propose a revised version of Zipf's law to incorporate the effect of size in the distribution function [15.

Zipf's law can be expressed as

$$
x(r)=x_{\max }(1+r)^{-\beta}
$$

in which $x$ may represent $D_{i}$ or $T_{i}$, depending on the variable to be fitted, and $r$ stands for the decreasing rank of $x$. It is a well known fact that the values of $\beta$ in the distributions of word frequency and city population approximate 1 14, 16. In data analysis, we randomly select a day (Mar. 1, 2013) and fit Eq 7 to hourly-based distributions of $D_{i}$ and $T_{i}$ for each of the top 1,000 forums. We find that: 1) The variance of $\beta$ is very small across hours, and 2) the maximum value of $x$ scales to system size (the number of threads $N$ ), satisfying

$$
x_{\max } \sim N^{\eta} \quad(\eta>\beta)
$$


Eq 8 implies that, the competition between threads for users $\left(D_{i}\right)$ and clicks $\left(T_{i}\right)$ becomes stronger as system size increases. As a result, the resources will concentrate to a small amount of threads, or, in some extreme cases, to the most popular thread. By putting together Eq.7 and Eq, 8 we have

$$
x(r)=x_{\max }(1+r)^{-\beta} \approx x_{\max } r^{-\beta} \approx N^{\eta} r^{-\beta} .
$$

Eq 9 is a revised version of Zipf's law in which the coefficient $N^{\eta}$ changes with system size. For relevant discussions see 17, 18. Meanwhile, combining Eq,7 and Eq,8 also gives

$$
x(r) \approx N^{\eta}(1+r)^{-\beta}=\left(N^{-\frac{\eta}{\beta}}+N^{-\frac{\eta}{\beta}} r\right)^{-\beta} .
$$

We define $k=\frac{r}{N}$ and get

$$
x(k) \approx\left(x_{\max }^{-\frac{1}{\beta}}+k N^{1-\frac{\eta}{\beta}}\right)^{-\beta} .
$$

Eq11 keeps both the information of the maximum value $X_{\max }$ and the size of system $N$ in the equation thus is doing better in fitting data. Therefore, in data analysis we use Eq11 instead of Eq 9 to estimate the empirical values of $\eta$ and $\beta$.

In Fig 3 we compare the revised version of Zipf's law against the traditional form of Zipf's law 1920 . Panel A shows that, the revised Zipf's law captures the deviation of the maximum values from the straightline prediction given by Zipf's law in the upper-left corner of the rank-ordered curve (Fig/3A). Panel B and $\mathrm{C}$ in Fig 3 compare the D statistic of the Kolmogorov-Smirnov test (the maximum of y values) between two models on an example data set, the distribution of Ti of the forum "EXO" (a Korean band) in the first hour of Feb. 8, 2013. The value of D statistic is 0.57 for Zipf's law (red curve) and 0.36 for the revised Zipf's law (green line), respectively. As a smaller D statistic means a better fit, we can conclude that the revised Zipf's law is a better distribution model. Panel D F shows that this conclusion can be generalized to all the studied 1,000 forums.

The revised Zipf's law not only provides a better fit of data, but more importantly, it also explains the scaling properties of online systems $(\mathrm{Eq}, 4$ and $\mathrm{Eq}, 5$. From Eq,9, we can derive the scaling relationship between system size $N$ and the sum of element values $W$ as

$$
W=\int_{1}^{N} x(r) d r \approx \int_{1}^{N} N^{\eta} r^{-\beta} d r=N^{\eta} \frac{1}{1-\beta}\left(N^{1-\beta}-1\right) \approx \frac{1}{1-\beta} N^{1+\eta-\beta}
$$

$\mathrm{Eq} 12$ connects the parameter of distribution with the parameter that shapes the growth dynamics. Using this function, we can predict the relative growth rates of $P V$ and $U V$ with retrospect to system size $N$ (which are $\theta_{2}$ in Eq4 and $\theta_{3}$ in Eq.5. respectively) by analyzing the hourly distributions of $T_{i}$ and $D_{i}$, respectively. After we obtain the theoretical values of $\theta_{3}$ and $\theta_{2}$, we can further predict the value of $\theta_{1}$ according to Eq,6, which now can also be expressed as

$$
\theta_{1}=\frac{1+\eta_{T}-\beta_{T}}{1+\eta_{D}-\beta_{D}}
$$

Fig 4 shows the empirical evidence supporting the connection between distribution and growth. Panel $\mathrm{A}$ and $\mathrm{B}$ show that the revised Zipf's law fits the distribution of dissipation $\left(D_{i}\right)$ and passing-through traffic $\left(T_{i}\right)$ in different hours very well. Panel C shows the scaling of users $(U V)$ and clicks $(P V)$ against the number of threads $(N)$. Panel $\mathrm{D}$ shows the scaling of the maximum values $D_{\max }$ and $T_{\max }$ with $N$. These two panels illustrate that the both the sum and the maximum value of variables scale with system size, as predicted by Eq 8 and Eq 12 . Panel E shows the scaling between $P V$ and $U V$ as given by Eq 3 . 

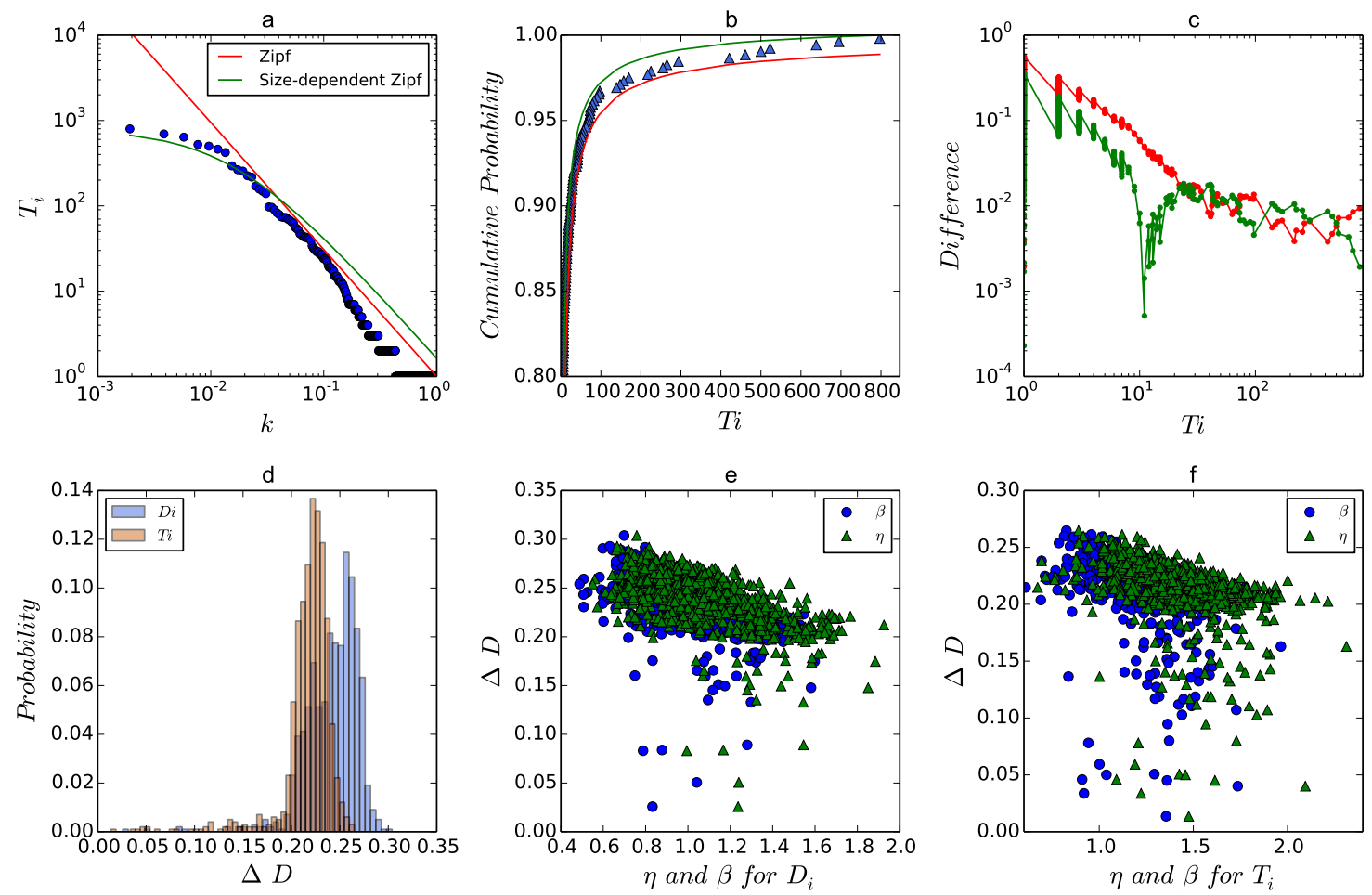

Figure 3. The comparison between Zipf's law (Eq7) and the revised Zipf's law (Eq11). In Panel A the blue circles show the distribution of $T_{i}$ of the forum "EXO" (a Korean band) in the first hour of Feb. 8, 2013. The fitting of Zipf's law is shown in red line and the fitting of the revised Zipf's law is shown in green line. In these curves $\beta=1.49$ and $\eta=1.44$. Panel B compares the cumulative distribution of $T_{i}$ between two models. Panel C compares the D statistic of the Kolmogorov-Smirnov test (the maximum of y values) between two models. In particular, the value of $\mathrm{D}$ statistic is 0.57 for Zipf's law (red curve) and 0.36 for the revised Zipf's law (green line), respectively. As a smaller D statistic means a better fit, we can conclude that revised Zipf's law is a better distribution model. In Panel D we define $\Delta D$ as the difference between the D-statistics of Zipf's law and the revised Zipf's law. If this quantity is greater than 0, it means that revised Zipf's law has the smaller D-statistics and thus is a better model. It is observed that the average values of $\Delta D$ for $D_{i}$ (blue) and $T_{i}$ (chocolate) over 24 hours are always greater than 0 across all the studied 1,000 forums. The mean values and standard deviations of these two distributions are 0.24 and 0.03 for $D_{i}$, and are 0.22 and 0.03 for $T_{i}$, respectively. Panel $\mathrm{E}$ and $\mathrm{F}$ show that the values of $\Delta D$ do not change dramatically with the values of $\beta$ and $\eta$. This means that $\mathrm{D}$ is a stable indicator for the comparison of fitting performance between the two models.

As discussed in our previous analysis, this is the consequence of the scaling relationships demonstrated in Panel D. Finally, Panel F shows that the theoretical prediction of $\theta_{1}$ given by Eq 13 (the red line) is supported by empirical data (the green dots). In sum, our analysis on the connection between distribution and growth allows us to predict the scaling functions of online systems exhibited in long-term evolution by analyzing hourly distributions. 

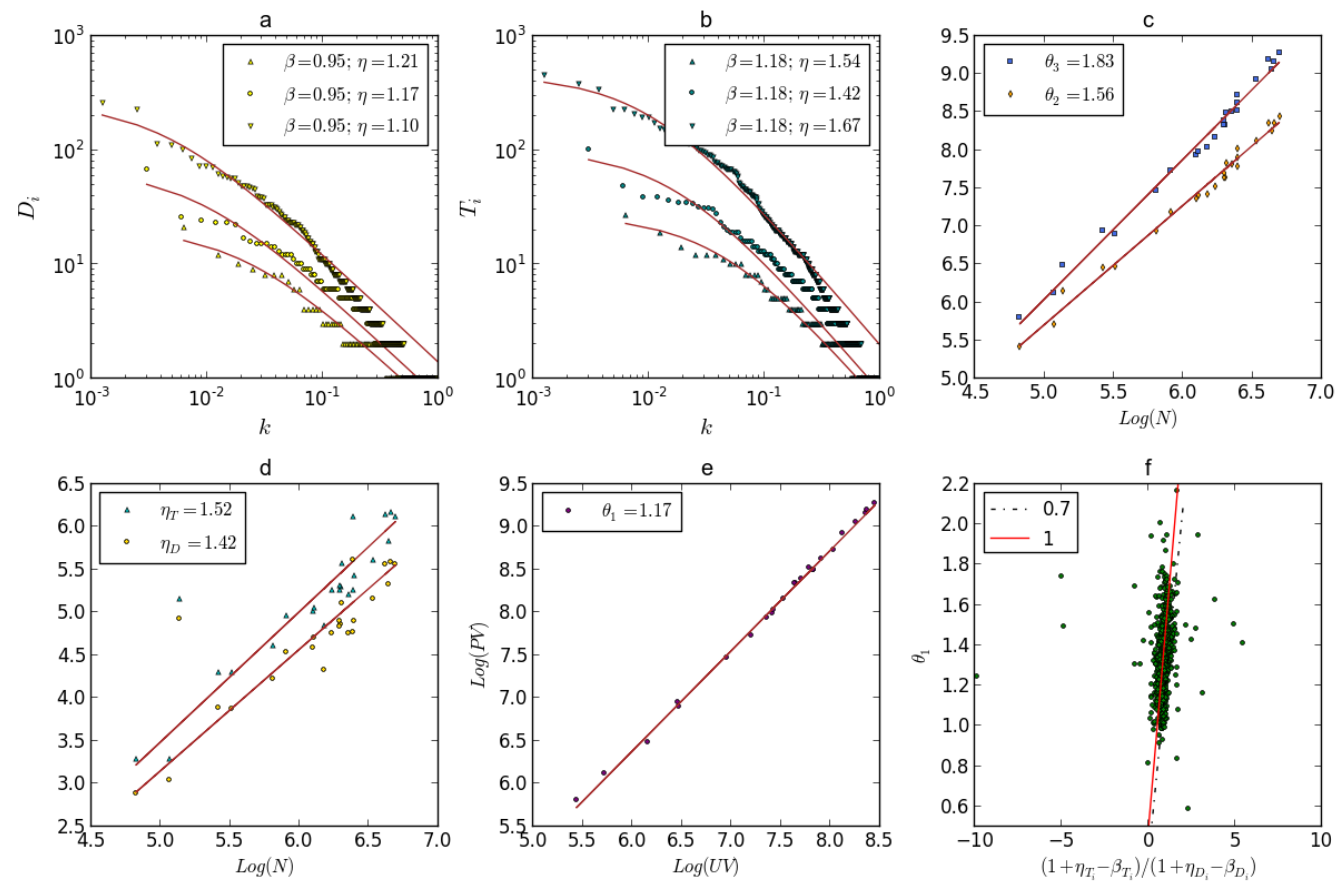

Figure 4. From size-depend Zipf's law to the scaling functions of forums. Panel A $\sim$ E are shown in a $\log -\log$ scale. Panel A and B show the rank-ordered curves of $D_{i}$ and $T_{i}$ in three hours (the 5 th, the 7th, and the 22nd hour) of the forum "EXO" on Feb. 8, 2013, respectively. The $x$ axis is the normalized decreasing rank (i.e., $k$ in Eq,11) and the $y$ axis shows the values of $D_{i}$ (or $T_{i}$ ). In both panels the value of $\beta$ (see Eq 11) is fixed to be the mean value over 24 hours, whereas the values of $\eta$ are estimated from the hourly data sets. Panel $\mathrm{C}$ shows the increase of $U V$ and $P V$ with the number of nodes (threads) and Panel D shows the increase of $D_{\max }$ and $T_{\max }$ with the number of nodes. These two panels illustrate that the both the sum of variables and the maximum of variables increase with system size, as predicted by Eq 8 and Eq, 12 , Panel E shows the scaling between $P V$ and $U V$ as given by Eq 3 As discussed in our previous analysis, this is the consequence of the scaling relationships demonstrated in Panel D. Panel F shows that the theoretical prediction of $\theta_{1}$ given by $\mathrm{Eq} 13$ (the red line) is supported by empirical data (the green dots). We also plot the regression line (the black, dotted line) to guide the eye. In all these panels, the orthogonal regression is used to fit data.

\section{The law of dissipation}

By replacing $x$ in Eq 9 with $D_{i}$ and $T_{i}$ we recover the revised Zipf's law of users and clicks on threads as

$$
D_{i}=N^{\eta_{D}} r^{-\beta_{D}}
$$

and

$$
T_{i}=N^{\eta_{T}} r^{-\beta_{T}}
$$


In Eq 14 and Eq15, $r$ is the decreasing rank of threads in terms of received users $\left(D_{i}\right)$ and clicks $\left(T_{i}\right)$, respectively. If we reasonably assume that these two ranks are equal or at least similar to each other, we will find that the distributions of users and clicks are connected, giving the law of dissipation

$$
D_{i}=T_{i}^{\gamma}
$$

Putting together Eq14 16 , we have

$$
\gamma=\frac{\beta_{D}}{\beta_{T}}=\frac{\eta_{D}}{\eta_{T}} .
$$

Eq 17 allows us to predict the law of dissipation from the distribution of $D_{i}$ and $T_{i}$.

Fig 5 shows the empirical evidence supporting the law of dissipation and its connection with the distributions of $D_{i}$ and $T_{i}$. Panel A shows the relationships between $D_{i}$ and $T_{i}$ in three hours (i.e., the 5th, the 7th, and the 22nd hour) on the forum "EXO" on Feb. 8, 2013. We observe that $D_{i}$ changes with $T_{i}$ linearly in a log-log scale, supporting our assumption on the power-law relationship between these two variables. In Panel B we show the distributions of the parameters fitted from the studied 1,000 forums using Eq16. The mean value of $\gamma$ is 0.68 and the mean value of $R^{2}$ is 0.82 . This shows that the law of dissipation exists widely in the studies forums. In Panel C and D we observe the linearly dependency of $\gamma$ on the division between the parameters of the distribution of $D_{i}$ and $T_{i}$, as predicted by Eq 17. All this is evidence that our derivations are supported by empirical analysis.

In flow networks, if $\gamma$ is greater than 1 , the dissipation of flow through nodes $\left(D_{i}\right)$ will increase faster than the traffic passing through nodes $\left(T_{i}\right)$. Thus nodes carrying large amounts of traffic will dissipate much more flow than nodes carrying small amounts of traffic. In other words, the dissipation of flow aggregates on high-traffic nodes. This pattern corresponds to a star-like topology of flow networks 2122 . On the contrary, if $\gamma$ is smaller than 1, the dissipation of flow is accomplished by many low-traffic nodes. This corresponds to a chain-like topology of flow networks [21 22]. To summarize, the finding of the law of dissipation with an exponent $\gamma$ smaller than 1 (the mean value of $\gamma$ is 0.68 according to Fig.5B ) in the current study shows that the transportation of attention flow on the studied forums forms a chain-like structure. Compared to the star-like topology, this type of structure is less efficient in dissipating users out of the systems, or, from another perspective, it does a good job in remaining users in the systems [23]. Therefore, $1 / \gamma$ can be used as an indicator for the attractiveness of forums. This indicator is invariant of system size thus it allows us to compare the attractiveness between Web forums across scales.

So far we have discussed two network-level indicators, $\theta_{1}$ and $1 / \gamma$. The former reflects the production efficiency of clicks in forums measured in a long time period (two months in our dataset) and the latter is related with the attractiveness of forums measured in a short time period (one hour in our dataset). As these two variables characterize different behaviors of forums, we can construct a two-dimensional feature space and compare the studied forums within this space. Fig $5 \mathrm{E} \sim \mathrm{F}$ give the distribution of 20 forum categories in the $\theta_{1}$ vs. $1 / \gamma$ space. Each circle corresponds to a category of forums. These categories are constructed by human labeling based on forum content. The size of purple circles reflects the number of forums in the category and the size of blue circles shows the average size of forums. After removing some forums due to a lack of labels, the remained data set includes 805 forums. We observe that $\theta_{1}$ and $1 / \gamma$ are positively correlated. This is not surprising because when $\gamma$ is small (or, when $1 / \gamma$ is large), the attention network has a chain-like structure which will remain users within the system for a longer period of time, leading to more clicks as well as a large $\theta_{1}$ in the long term.

Beside theoretical inspirations, the $\theta_{1}$ vs. $1 / \gamma$ space also provides insight into the management of Web forums. As can be seen from Fig $5 \mathrm{E} \sim \mathrm{F}$, the categories "Art" and "TV programs" locate at the lower-left corner of the space, suffering from unattractiveness and low production efficiency of clicks. On the contrary, the categories "Romantic" and "Cities" are both attractive and productive. This means that Baidu Tieba users seek for online interactions that may be turned into offline opportunities. Another interesting observation is the hight clickstream productivity of the "Female" category, which implies the 

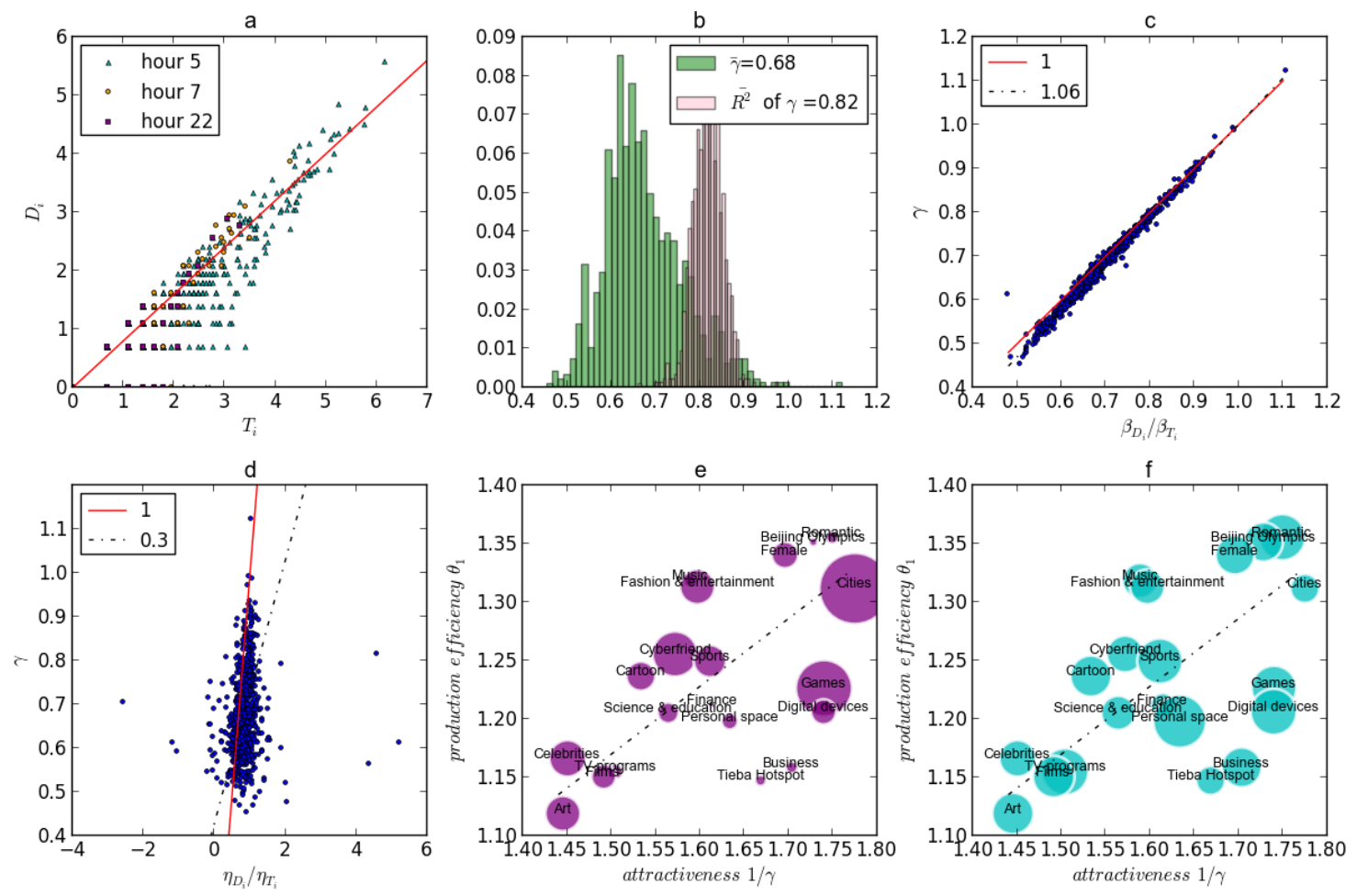

Figure 5. The law of dissipation and its connections with scaling functions. Panel A shows the relationships between $D_{i}$ and $T_{i}$ in three hours (the 5 th, the 7 th, and the $22 \mathrm{nd}$ hour) of the forum "EXO" on Feb. 8, 2013. The average value of $\gamma$ in 24 hours, which equals 0.79 , is plotted as red line for comparison. Panel B gives the distribution of the hourly-averaged value of $\gamma$ and $R^{2}$ across 990 forums (the rest 10 forums are removed due to a lack of data that allows a validated estimation of $\gamma$ ). Panel $\mathrm{C}$ shows the prediction of $\gamma$ from $\beta$ s and Panel D show the prediction of $\gamma$ from $\eta \mathrm{s}$. In these two subplots we both give the regression line fitted from data points (the black, dotted lines) and also the theoretical line (the red lines) predicted by Eq.17. In Panel E and Panel $\mathrm{F}$ we show the distribution of forum categories in the feature space. The $x$ axis shows the attractiveness $1 / \gamma$ and the $y$ axis shows the production efficiency $\theta_{1}$. Each circle corresponds to a category of forum. The size of purple circles reflects the number of forums in the category and the size of blue circles shows the average size of forums within the category. In Panel $\mathrm{E}$ and Panel $\mathrm{F}$ we also plot the regression line fitted from the plotted bubbles to show the positive relationship between $1 / \gamma$ and $\theta_{1}$.

existence of a large portion of female users. This observation is supported by the website statistics provided by Alexa (www.alexa.com), which suggests that the percentage of females in Tieba users is much higher than the average level across the entire Web . 


\section{Discussion and Conclusion}

It is well acknowledged that human beings are experiencing an age of information explosion, in which attention becomes a very scarce resource. As a consequence, there is a surge of interest on attention dynamics in recent years 3,7]. Inspired by [3, 4], we model the collective browsing behavior of millions of users by attention networks and discuss the dynamics of attention from a network perspective. We find that time-invariant flow structures are preserved by attention networks during their evolution. As a result, the studied online systems exhibit various scaling relationships between threads, users, and clicks $\left[15,21,24\right.$. We suggest that the exponent $\theta_{1}$ in the scaling relationship between clicks and users can be used as a measure of the production efficiency of forums in obtaining user attention. We also introduce a novel regularity, the dissipation law, to quantify the transportation of users and clicks between nodes. The parameter $\gamma$ in the dissipation law is related with the attractiveness of forums and thus has an effect on the production efficiency $\theta_{1}$. Finally, we demonstrate how to put these two indicators together to obtain management insights.

\section{Acknowledgments}

L.W. acknowledges the financial support for this work from the National Science Foundation, grant number 1210856. CJ.W. acknowledges the financial support for this work from the National Social Science Foundation of China, grant number 15CXW017, the China Postdoctoral Science Foundation, grant number 2015M571722, and the Fundamental Research Funds for the Central Universities, grant number 2062015008 .

\section{References}

1. Simon HA (1973) Applying information technology to organization design. Public Administration Review 33: 268-278.

2. Barabasi AL (2005) The origin of bursts and heavy tails in human dynamics. Nature 435: $207-211$.

3. Wu F, Huberman BA (2007) Novelty and collective attention. Proceedings of the National Academy of Sciences 104: 17599-17601.

4. Cattuto C, Barrat A, Baldassarri A, Schehr G, Loreto V (2009) Collective dynamics of social annotation. Proceedings of the National Academy of Sciences 106: 10511-10515.

5. Weng L, Flammini A, Vespignani A, Menczer F (2012) Competition among memes in a world with limited attention. Scientific Reports 2.

6. Lehmann J, Gonçalves B, Ramasco JJ, Cattuto C (2012) Dynamical classes of collective attention in twitter. In: Proceedings of the 21st international conference on World Wide Web. ACM, pp. 251-260.

7. Sasahara K, Hirata Y, Toyoda M, Kitsuregawa M, Aihara K (2013) Quantifying collective attention from tweet stream. PloS one 8: e61823.

8. Blank A, Solomon S (2000) Power laws in cities population, financial markets and internet sites (scaling in systems with a variable number of components). Physica A: Statistical Mechanics and its Applications 287: 279-288.

9. Dreyer O (2001) Allometric scaling and central source systems. Physical review letters 87: 038101. 
10. West GB, Brown JH, Enquist BJ (1997) A general model for the origin of allometric scaling laws in biology. Science 276: 122-126.

11. Banavar JR, Maritan A, Rinaldo A (1999) Size and form in efficient transportation networks. Nature 399: 130-132.

12. Wang J, Li Q, Chen YP (2010) User comments for news recommendation in social media. In: Proceedings of the 33rd international ACM SIGIR conference on Research and development in information retrieval. ACM, pp. 881-882.

13. Bollen J, Van de Sompel H, Hagberg A, Bettencourt L, Chute R, et al. (2009) Clickstream data yields high-resolution maps of science. PLoS One 4: e4803.

14. Zipf GK (1949) Human behavior and the principle of least effort. Addison-Wesley Press, Massachusetts.

15. Wu L, Zhang J (2011) Accelerating growth and size-dependent distribution of human online activities. Physical Review E 84: 026113.

16. Batty M (2008) The size, scale, and shape of cities. science 319: 769-771.

17. Batty M (2006) Rank clocks. Nature 444: 592-596.

18. Cristelli M, Batty M, Pietronero L (2012) There is more than a power law in zipf. Scientific reports 2 .

19. Eeckhout J (2004) Gibrat's law for (all) cities. American Economic Review : 1429-1451.

20. Newman ME (2005) Power laws, pareto distributions and zipf's law. Contemporary physics 46 : $323-351$.

21. Zhang J, Wu L (2013) Allometry and dissipation of ecological flow networks. PloS one 8: e72525.

22. Wu L, Zhang J (2013) The decentralized flow structure of clickstreams on the web. The European Physical Journal B 86: 1-6.

23. Wu L, Zhang J, Zhao M (2014) The metabolism and growth of web forums. PloS one 9: e102646.

24. Wu L (2011) The accelerating growth of online tagging systems. The European Physical Journal B-Condensed Matter and Complex Systems 83: 283-287. 